Ebisu Ebisu

Études japonaises Études japonaises

47 | printemps-été 2012

Catastrophes du 11 mars 2011, désastre de

Fukushima : fractures et émergences

\title{
Vivre, vivre au Japon
}

Living and Being Alive in Japan

日本に生る

Manuel Tardits

(2) OpenEdition

Journals

Édition électronique

URL : http://journals.openedition.org/ebisu/468

DOI : 10.4000/ebisu.468

ISSN : 2189-1893

Éditeur :

Institut français de recherche sur le Japon (UMIFRE 19 MAEE-CNRS), Maison franco-japonaise

Édition imprimée

Date de publication : 1 juin 2012

Pagination : 223-227

ISSN : 1340-3656

Référence électronique

Manuel Tardits, «Vivre, vivre au Japon », Ebisu [En ligne], 47 | printemps-été 2012, mis en ligne le 03 avril 2014, consulté le 19 avril 2019. URL : http://journals.openedition.org/ebisu/468 ; DOI : 10.4000/ ebisu. 468

(C) Institut français de recherche sur le Japon à la Maison franco-japonaise 


\title{
Vivre, vivre au Japon
}

\author{
Manuel TARDITS
}

La forte secousse qui, parmi les centaines d'autres répliques, a de nouveau ébranlé tout l'est du Tōhoku le jeudi 7 avril 2011, près d'un mois après le séisme et le tsunami du 11 mars, n'a eu qu'un seul mérite, si l'on ose dire : replacer le débat dans la perspective plus large de l'écologie et non celle primordiale mais parfois trop étroite du seul nucléaire.

Dans un courrier de lecteur publié dans Le Monde quelques jours seulement après le tsunami, son auteur établissait une différence significative entre la catastrophe de la centrale nucléaire de Fukushima censée résulter d'une situation créée par l'homme et les autres d'origines naturelles. Nous n'adhérons pas pleinement à cette opinion. Les problèmes rencontrés par la centrale accablent certes l'opérateur Tepco, mettent également en cause le fonctionnement de l'agence japonaise de contrôle et questionnent plus généralement l'option nucléaire. Pourtant, nombre de ces faits inacceptables par les citoyens du Japon ne doivent pas faire oublier que toutes ces catastrophes sans exceptions sont intimement liées à la présence de l'homme. Les ravages côtiers, s'ils n'avaient touché qu'un espace dépeuplé, resteraient surtout un sujet de préoccupation scientifique et écologique. Le caractère si meurtrier du tsunami et non du séisme est lié à l'amplitude imprévue de

Architecte DPLG (France), cofondateur de l'agence d'architectes Mikan, diplômé d'un master en ingénierie de l'université de Tokyo, sous-directeur de l'école ICS College of Arts (Tokyo), Chevalier dans l'Ordre des Arts et des Lettres. 
la vague dans cette région Pacifique pourtant souvent soumise par le passé à des raz-de-marée très meurtriers mais de moindre ampleur. En d'autres mots, il était notoire que les villes et les villages côtiers étaient exposés, mais on pensait généralement les mesures de sécurité suffisantes (jetées, briselames, murs de protection, annonce rapide du risque par le réseau de surveillance, zones de repli) pour prévenir non pas d'éventuelles destructions, mais préserver la majeure partie des vies humaines. Un principe similaire est d'ailleurs à l'œuvre dans les calculs structurels qui régissent la construction de tout bâtiment au Japon : à défaut de pouvoir empêcher tous les dommages, la sévérité des règlements cherche à minimiser les pertes en vies humaines en limitant les effondrements brusques. Le 11 mars, la protection et la prévention ont été prises en défaut de façon dramatique : là est le réel problème et le premier sujet d'angoisse et de perte de confiance. De nature différente, un autre sujet d'étonnement et de stupeur fut de constater la vitesse et la précision anticipatrices des annonces médiatiques et leur peu d'effet pour les victimes. Nous-mêmes à Tokyo, après avoir été fortement ébranlés, pouvions regarder la chronique de ces morts annoncés dans les minutes suivant la secousse. Pour qui y avait accès, des chaînes de télévision retransmettaient en direct l'événement et surtout montrait un affichage quasi immédiat sur les écrans de la hauteur des vagues prévues et de leurs horaires d'arrivée localité par localité. Cette prévision précédait souvent de plus de 10 minutes la montée des eaux selon les villes, pourtant ces images n’atteignaient pas les gens concernés directement par le danger.

Pour en revenir donc au séisme suivi d'un léger tsunami du jeudi 7 avril, ce dernier a souligné mais de manière moins létale le caractère récurrent des risques naturels au Japon. Le 11 mars n'est hélas qu'un drame qui en suit et en précède d'autres. L'imminence souvent annoncée d'un séisme majeur, le Big One, à Tokyo est dans tous les esprits. Comme dans nombre d'autres régions du globe, le Japon est soumis à ces aléas de la tectonique des plaques qui échappent encore à la prévision fiable. Que faire donc ? Partir, sombrer dans le fatalisme? Arrêtons de revenir aux poncifs sur le sentiment du caractère éphémère des choses hérité du bouddhisme et d'une culture locale acceptée de l'impermanence imprégnée par la dangerosité d'un climat et d'une géographie, pour expliquer le comportement calme et civique des Japonais. Non seulement des centaines de milliers d'étrangers vivent ici dans les mêmes conditions, qui ne possèdent pas forcément cette 
culture et respectent des principes similaires, mais surtout les sentiments des Japonais sont marqués de beaucoup plus d'ambiguïté. À Tokyo, si chacun est conscient qu'à tout moment une catastrophe naturelle majeure peut frapper et remettre nos existences en question, l'hédonisme existe ainsi qu'une certaine foi en l'aptitude de l'organisation et de la technologie d'aujourd'hui à nous aider et même nous sauver. Avons-nous peur ? Oui, mais nous ne pouvons qu'édulcorer la pensée quotidienne du risque. Le contraire est simplement impensable à gérer psychologiquement même si certaines précautions au quotidien sont de mise. Naît alors une sorte de trilogie des sentiments : crainte, acceptation du risque mais aussi et surtout opiniâtreté à survivre et à rechercher des solutions pour gérer le caractère inéluctable et encore imprévisible à l'heure actuelle des catastrophes. Ce troisième terme est d'ailleurs la seule alternative valable car il implique de faire face en se tournant vers le futur. À cet endroit, dépasser les mesures de prévention ponctuelles et de gestion postérieures du quotidien d'après la catastrophe pour leur adjoindre une vision écologique plus large et renouvelée s’impose.

Nul ne peut encore dire comment le Japon, sa population et ses élites, vont réagir une nouvelle fois au risque sismique et à ses implications écologiques. Plusieurs grandes questions sont toutefois posées ou reposées. La première concerne la pertinence de notre civilisation basée sur le progrès de la technologie. Si les progrès dans les techniques de construction et la sévérité accrue des réglementations réduisent les risques sans les supprimer (peu de dégâts constatés malgré la force des secousses ce qui rassure tout de même), dans le cas du nucléaire cette même option technologique les augmente. Au final, la technologie n'a d'ailleurs su ni empêcher ni prévenir la lame d'eau. Dans certains villages, les habitants disparus se sont même crus, à tort, protégés par les ouvrages de génie civil. Nous avons une nouvelle fois, comme à Kobe, pensé que nos progrès nous sauveraient. Quant à la sûreté nucléaire, peut-elle être vraiment assurée, quelles que soient les assurances des autorités, dans un pays touché périodiquement par des incidents naturels majeurs? Plus encore qu'en France, la prise de ce risque doit faire l'objet d'un débat démocratique. En d'autres mots, au-delà de l'émotion, il s'agit de comparer les avantages (il y en a) et les inconvénients de la filière énergétique nucléaire. 
La seconde question qui se pose concerne nos modèles de développement urbains et sociétaux. L'hypertrophie des centres urbains, peu touchés cette fois-ci (par respect pour les victimes du Tōhoku, on n'ose pas encore trop parler de Tokyo, conurbation de 37 millions d'habitants qui accueille près d'un Japonais sur trois, si l'épicentre avait été plus proche), doit-elle être remise en question ? L'arrêt immédiat le 11 mars de tout le réseau ferré de la capitale bloquant en milieu d'après-midi des millions de gens sur leur lieu de travail, les enfants dans leurs écoles et la déficience de nombreux réseaux de communication téléphoniques laissant la grande masse des gens dans la quasi impossibilité de se joindre (à l'exception du réseau fixe, surchargé mais resté opérationnel), montrent à l'évidence la difficulté de gérer une situation de crise dans une telle concentration humaine. Répartir les risques, éviter l'embolie du système par la répartition du peuplement et des centres névralgiques, telles sont les questions récurrentes. Des modèles alternatifs de dédensification urbaine sont-ils envisageables et viables dans un pays qui entame une baisse démographique sans précédent et dont les campagnes se vident au profit des villes. Nos modèles consumériste et énergétique sont également en cause. La réduction drastique de notre consommation électrique afin de soulager la déficience momentanée (quelques mois quand même !) des capacités de production d'énergie dans la région du Kantō est-elle possible durablement et compatible avec nos habitudes récentes mais exigeantes d'usage et de confort ? Le civisme ambiant répond par l'affirmative, mais tout le monde pense déjà aux grosses chaleurs torrides de l'été et à la voracité des systèmes de réfrigération peu compatibles avec l'économie d'énergie. On peut décider d'éteindre façades et néons pour plonger les quartiers commerciaux dans une pénombre nocturne quelque peu inquiétante, mais supporter la chaleur dans des bureaux ou des maisons fermées en août requiert des efforts autrement plus pénibles. Consommer moins pour produire moins, telle est la question.

Oui, nous sommes prêts à vivre ici, sans fatalisme quoique en acceptant les risques naturels inhérents à la vie japonaise. Oui, les gens retourneront sans doute vivre dans les zones dévastées par le tsunami (pour l'instant aucune autorité n'a formellement déclaré non aedificandi toutes les zones inondables). Oui, à l'exception des aires polluées pour des dizaines d'années, nous continuerons de vivre à Tokyo près de la centrale nucléaire de Fukushima mais, au-delà, notre société voudra-t-elle planifier sa propre 
réduction : revoir ses villes, ses options techno-industrielles et énergétiques, un style de vie consumériste encouragé depuis le bond en avant de l'aprèsguerre? Saurons-nous faire ce choix d'une nouvelle écologie que nous avons évité jusqu'à maintenant? Quel sera le prix à payer pour aimer vivre dans le Tōhoku, à Tokyo, ou même simplement vivre?

Avril 2011 